\title{
Influência do tempo e meio de armazenamento na resistência adesiva e microinfiltração de sistemas adesivos
}

\author{
Influence of time and medium of storage on the bond strength and microleakage \\ of adhesive systems
}

\begin{abstract}
Leonardo Vieira de Oliveira, ${ }^{1}$ Maíra do Prado, ${ }^{2}$ Carlos Tadeu dos Santos Dias, ${ }^{3}$ Adriano Nóbrega de Castro, ${ }^{4}$ Gisele Damiana da Silveira Pereira, ${ }^{5}$ José Roberto Louvadino
${ }^{1}$ Departamento de Clínica Odontológica, Faculdade de Odontologia de Piracicaba, Universidade Estadual de Campinas, Piracicaba, SP, Brasil

${ }^{2}$ Faculdade de Odontologia, Centro de Saúde, Universidade Veiga de Almeida, Rio de Janeiro, RJ, Brasil

${ }^{3}$ Departamento de Matemática e Estatística, Escola Superior de Agronomia Luiz de Queiroz, São Paulo, SP, Brasil

${ }^{4}$ Especialização em Radiologia, Instituto Nacional de Ciências Odontológicas, Rio de Janeiro, RJ, Brasil

${ }^{5}$ Departamento de Clínica Odontológica, Faculdade de Odontologia, Universidade Federal do Rio de Janeiro, Rio de Janeiro, RJ, Brasil

- Os autores declaram que não há conflito de interesse.

\section{Resumo}

Objetivo: avaliar a influência do tempo e meio de armazenamento na resistência à tração de dois sistemas adesivos após diferentes períodos de armazenamento. Material e Métodos: oitenta incisivos bovinos foram selecionados e aleatoriamente divididos em oito grupos $(n=10)$ de acordo com o sistema adesivo, Adper Scotchbond Multi-Purpose (ASMP) e Clearfil SE Bond (CLSEB), meio de armazenagem [água (A) ou solução de Desmineralização/Remineralização (DES/RE)] e tempo (7 ou 30 dias). Após a armazenagem, as amostras foram submetidas a ensaio mecânico e para análise "in vitro" de resistência de união (RU). A nanoinfiltração da camada híbrida foi avaliada através de Microscopia Eletrônica de Varredura (MEV) e seus constituintes foram avaliados por microanálise (EDS). Os valores de RU foram analisados estatisticamente por ANOVA ( $p<$ 0,05). Resultados: os valores de RU do ASBMP foram: A7dias: 14,86 $\pm 5,64$; A30dias: 13,60 $\pm 3,57$; DES/RE7dias: $13,60 \pm 3,57$; DES/RE30dias: $13,24 \pm 4,19$. Para o CLSEB os valores de RU obtidos foram: A7dias: $13,16 \pm 5,26$; A30dias: 6,55 $\pm 1,82$; DES/RE7dias: 10,27 $\pm 3,87$; DES/RE30dias: 8,42 $\pm 1,69$. Conclusão: concluiu-se que o uso de solução DES/RE associada à imersão dos espécimes em solução aquosa de NaF $0,05 \%$ foi benéfica para a longevidade e integridade da camada híbrida ao longo do tempo. Palavras-chave: Adesivos dentinários; Fluoreto de sódio; Infiltração dentária; Restauração dentária permanente.

\section{ABSTRACT}

Objective: the aim of this study was to evaluate the influence of time and medium of storage in the tensile strength of two adhesive systems after different periods of storage. Material and Methods: eighty bovine incisors were selected and randomly divided into eight groups $(n=10)$ according to the adhesive system, which are as follows: Adper Scotchbond Multi-Purpose (ASBMP) and Clearfil SE Bond (CLSEB), medium of storage [water (A) or demineralization / remineralization solution (DES/RE)] and time of storage (7 or 30 days). After storage, the samples were subjected to mechanical testing to analyze, "in vitro," the bond strength (BS). The nanoleakage of the hybrid layer was evaluated using scanning electron microscopy (SEM), and its constituents have been assessed by energy-dispersive $X$-ray spectroscopy (EDS) microanalysis. The BS values were statistically analyzed using ANOVA ( $p<0.05)$. Results: the BS values of ASBMP were A7 days: $14.86 \pm 5.64 ;$ A30 days: $13.60 \pm 3.57 ; D E S / R E 7$ days: $13.60 \pm 3.57$; DES/RE30 days: $13.24 \pm$ 4.19. For CLSEB the values obtained were A7 days: $13.16 \pm 5.26$; A30 days: $6.55 \pm 1.82$; DES/RE7days: $10.27 \pm 3.87$; DES/RE30days: $8.42 \pm 1.69$. Conclusion: the zirconia ceramics did not present compositional differences, but the morphology and size of crystals varied depending on the type of zirconia.
\end{abstract}

Keywords: Dental leakage; Dentin-bonding agents; Sodium fluoride; Dental restoration permanent.

\section{Introdução}

O processo de degradação das restaurações ocorre a partir da microinfiltração nos poros presentes na interface adesiva defeituosa da camada híbrida, que ocorre devido à presença de uma camada de 20-100 nm de dentina desmineralizada não infiltrada pelos monômeros, ${ }^{1}$ que possibilita o livre trânsito de fluido que é capaz de diluir os monômeros não reagidos, além de causar hidrólise e plastificação da rede polimérica que irá culminar com a perda de propriedades à longo prazo. ${ }^{2}$

Os sistemas adesivos, de maneira geral, apresentam como principais componentes: monômeros resinosos, iniciadores, solventes, inibidores e em alguns casos partículas de carga inorgânicas. Entretanto, a alteração dos percentuais e tipos destes componentes mudam significativamente as propriedades finais destes materiais, alterando, seu grau de conversão, resistência adesiva, resistência a hidrólise e índice de sorção. ${ }^{3}$

Além dos fatores intrínsecos referentes à composição dos materiais deve-se destacar que há aspectos ligados ao substrato dentinário e ao meio oral que influenciarão diretamente a degradação da camada híbrida adesiva. Estes aspectos incluem a degradação do HEMA, componente hidrofílico mais comum dos sistemas adesivos, por exemplo, que é mais ocorrente em meio básico e a existência de subprodutos da reação de condicionamento ácido que atacam as ligações éster do agente de união e são capazes de ativar a degradação local do colágeno pela ativação de enzimas colagenolíticas. ${ }^{2,4-7}$

Contudo, diante do fato de que a interface adesiva da camada híbrida apresenta-se como uma membrana semipermeável, que possibilita a entrada de fluído, e agentes deletérios conseguem penetrar na camada híbrida, é válida a postulação de que há a permeação de íons como cálcio, fosfato e flúor, que possibilitam a precipitação destes minerais que em sua forma cristalina poderiam preencher as porosidades e reduzir a permeabilidade destas áreas retardando a degradação do sistema adesivo. Desta forma, torna-se evidente a necessidade de avaliar a longevidade da união de sistemas adesivos aplicados na dentina em função da resistência à microtração após o armazenamento em água ou solução $\mathrm{DES} / \mathrm{RE}$, associado às soluções como o NaF.

\section{Material e Métodos}

Para a realização deste estudo foram utilizados um compósito restaurador micro-híbrido (TPH spectrum, Dentsply Ltda-Petró- 
polis, RJ,Brasil), dois sistemas adesivos, sendo um convencional de três passos (Adper Scotchbond Multi-Purpose, 3M do Brasil- Sumaré, SP, Brasil) e um autocondicionante (Clearfil SE Bond, Kuraray Medical-Okayama. Japão) de dois passos, ácido fosfórico 37\% (FGM Produtos Odontológicos Ltda, Joinville, SC, Brasil) e uma solução DES/RE e solução aquosa de NaF 0,05\% (Tabela 1).

Tabela 1. Composição das soluções de armazenamento

\begin{tabular}{|c|c|}
\hline Solução & Componentes \\
\hline $\begin{array}{l}\text { SOLUÇÃO } \\
\text { DESMINERALIZADORA }\end{array}$ & $\begin{array}{l}\text { - } 2,0 \mathrm{mM} \mathrm{Ca}(\mathrm{OH}) 2 ; \\
\text { - 2,0 mM H3PO4; } \\
\text { - } 75 \mathrm{mM} \mathrm{H} 3 \mathrm{CCOOH} ; \\
\text { - } \quad \text { Cristais de Timol. }\end{array}$ \\
\hline $\begin{array}{l}\text { SOLUÇÃO } \\
\text { REMINERALIZADORA }\end{array}$ & $\begin{array}{l}\text { - } 1,5 \mathrm{mM} \mathrm{Ca}(\mathrm{OH}) 2 ; \\
\text { - } 0,9 \mathrm{mM} \mathrm{H} 3 \mathrm{PO} 4 ; \quad \mathrm{pH} \mathrm{7,0} \\
\text { - } 150 \mathrm{mM} \mathrm{KCl} ; \\
\text { - } 20 \mathrm{mM} \text { Tampão Tris (hidroximetil) } \\
\text { aminometano. }\end{array}$ \\
\hline $\begin{array}{l}\text { SOLUÇÃO } \\
\text { FLUORETADA }\end{array}$ & - Fluoreto de Sódio (NaF) a 0,05\%. \\
\hline
\end{tabular}

A variável de resposta foi a resistência à microtração em três fatores: sistemas adesivos (Adper Scotchbond Multi-Purpose ou Clearfil SE Bond); período de armazenamento (7 ou 30 dias); solução de armazenamento (água destilada ou solução DES/RE associada à imersão dos espécimes em solução aquosa de $\mathrm{NaF}$ 0,05\%). A associação desses três níveis constituiu oito grupos ( $\mathrm{n}=$ 10) experimentais em um delineamento inteiramente ao acaso em esquema fatorial, como descrito na Tabela 2.

Tabela 2. Grupos experimentais para o ensaio de resistência à microtração

\begin{tabular}{l|l|l|l}
\hline Grupo & \multicolumn{1}{|c|}{$\begin{array}{c}\text { Sistema } \\
\text { adesivo }\end{array}$} & $\begin{array}{c}\text { Meio de } \\
\text { armazenamento }\end{array}$ & $\begin{array}{c}\text { Tempo de } \\
\text { estocagem }\end{array}$ \\
\hline 1 & ASBMP & Água & 7 dias \\
\hline 2 & ASBMP & Água & 30 dias \\
\hline 3 & ASBMP & $\begin{array}{l}\text { Solução DES/RE }+ \\
\text { NaF 0,05\% }\end{array}$ & 7 dias \\
\hline 4 & ASBMP & $\begin{array}{l}\text { Solução DES/RE }+ \\
\text { NaF 0,05\% }\end{array}$ & 30 dias \\
\hline 5 & CLSEB & Água & 7 dias \\
\hline 6 & CLSEB & Água & 30 dias \\
\hline 7 & CLSEB & $\begin{array}{l}\text { Solução DES/RE }+ \\
\text { NaF 0,05\% }\end{array}$ & 7 dias \\
\hline 8 & CLSEB & $\begin{array}{l}\text { Solução DES/RE }+ \\
\text { NaF 0,05\% }\end{array}$ & 30 dias \\
\hline
\end{tabular}

Foram utilizados oitenta incisivos bovinos hígidos armazenados por um período máximo de 1 mês em solução de timol 0,1\% a uma temperatura de $37^{\circ} \mathrm{C}$ até o início da limpeza das superfícies externas. Após a limpeza, os dentes foram armazenados em água destilada até o início da realização do preparo das superfícies.

A porção radicular foi separada da porção coronária através de uma secção perpendicular ao longo eixo do dente próximo à junção amelo-cementária. Posteriormente, a superfície palatina de todos os dentes foi removida através do desgaste com lixas de SiC 150 (BUEHLER Worldwide Headquarters 41 Waukegan Rd. P.O. Box 1Lake Bluff, IL 60044-1699, USA) acoplada em politriz mecânica (MaxiGrid Solotest, São Paulo, SP, Brasil) sob constante irrigação a água.

A superfície vestibular das amostras foram lixadas sequencialmente com lixas de SiC números 320 e 400 (BUEHLER Worldwide Headquarters 41 Waukegan Rd. P.O. Box 1Lake Bluff, IL 60044-1699, USA), em politriz elétrica giratória refrigerada a água até a obtenção de uma superfície plana em dentina com aproximadamente 2,5 mm de espessura. Em seguida, foi realizado o acabamento das superfícies em dentina através do desgaste manual com lixas de SiC úmidas (3M- 411Q 3M do Brasil- Sumaré, SP, Brasil) número 6008 durante 15s, proporcionando, desta forma, a padronização da espessura da "smear layer" e, posteriormente, lavados com jatos de água destilada e deionizada por 15 s permanecendo imersos em água destilada e deionizada por $24 \mathrm{~h}$ até o início dos procedimentos restauradores.

Os procedimentos restauradores se deram conforme os grupos experimentais listados abaixo (Tabela 2):

GRUPO 1- Sistema adesivo- Adper Scotchbond Multi-Purpose, Armazenamento-Água 7 dias.

GRUPO 2- Sistema adesivo- Adper Scotchbond Multi-Purpose, Armazenamento-Água 30 dias.

GRUPO 3- Sistema daesivo- Adper Scotchbond Multi-Purpose, Armazenamento- Solução DES/RE + NaF 0,05\% 7 dias.

GRUPO 4- Sistema adesivo- Adper Scotchbond Multi-Purpose, Armazenamento- Solução DES/RE + NaF 0,05\% 30 dias.

GRUPO 5- Sistema adesivo- Clearfil SE Bond, Armazenamento- Água 7 dias.

GRUPO 6- Sistema adesivo- Clearfil SE Bond, Armazenamento- Água 30 dias.

GRUPO 7- Sistema adesivo- Clearfil SE Bond, Armazenamento- Solução DES/RE + NaF 0,05\% 7 dias.

GRUPO 8- Sistema adesivo- Clearfil SE Bond, Armazenamento- Solução DES/RE + NaF 0,05\% 30 dias.

Imediatamente antes da aplicação dos tratamentos superficiais, o excesso de água foi removido por capilaridade com auxílio de papel absorvente (Melitta do Brasil- Ind. e Com. Ltda, Avaré, SP, Brasil). Feito isso, a etapa restauradora foi iniciada com o devido tratamento da dentina a depender do sistema adesivo utilizado.

Adper Scotchbond Multi-Purpose: foi realizado previamente o condicionamento ácido da dentina com ácido fosfórico a $37 \%$ por $15 \mathrm{~s}$. Em seguida, a superfície foi lavada abundantemente por 30 s e levemente seca com bolinha de algodão a fim de remover o excesso de água. . O "primer" do sistema Adper Scotchbond Multi-Purpose foi aplicado com auxílio de um pincel do tipo "microbrush” (FGM Produtos Odontológicos Ltda., Joinville, SC, Brasil) na dentina condicionada e levemente seco por $5 \mathrm{~s}$ a uma distância de $10 \mathrm{~cm}$. Posteriormente, foi aplicado o adesivo e fotopolimerizado por 10s com o aparelho Degulux Soft-Start (Degussa-Hüls AG- Geschãftsbereich Dental) com potência de aproximadamente $650 \mathrm{~mW} / \mathrm{cm}^{2}$ aferida constantemente em radiômetro digital (Cure Rite- Kerr- EOS). 
Clearfil SE Bond: o "primer" autocondicionante do sistema Clearfil SE Bond foi aplicado ativamente na dentina com auxílio de um pincel do tipo "microbrush". Foi aguardado por 20 s e, em seguida, secado com um tênue jato de ar por $5 \mathrm{~s}$ a uma distância de $10 \mathrm{~cm}$. Logo após, o adesivo foi aplicado por toda a superficie dentinária e fotoativado por 10s pelo aparelho fotopolimerizador Degulux Soft-Start, como recomendado pelo fabricante.

Após a aplicação dos respectivos sistemas adesivos, a resina composta TPH Spectrum, cor A2 foi aplicada primeiramente com 2,0 mm de espessura através de uma espátula metálica para resina composta (Duflex, SSWhite. Rio de Janeiro, RJ, Brasil) e posteriormente fotoativada por 40s pelo aparelho Degulux Soft-Start, no modo "soft-start". Posteriormente, foi adicionado um novo incremento com intuito de confeccionar um bloco retangular com aproximadamente $4,0 \mathrm{~mm}$ de altura, $8,0 \mathrm{~mm}$ de comprimento e 3,0 mm de largura com o mesmo tempo e modo de ativação inicial, seguido de uma fotopolimerização final adicional de 20 s nas suas porções mesial e distal em potência máxima. ${ }^{10}$ As amostras foram, então, submersas em água destilada, sem adição de antimicrobianos no qual permaneceram por $24 \mathrm{~h}$.

As coroas dentais foram individualmente fixadas pela porção palatina, em placas de acrílico, e seccionadas através de uma cortadeira metalográfica de precisão (ISOMET 1000, BUEHLER Ltda, Lake Buff, IL, USA) com um disco diamantado (Extec Corp., Enfield, CT, USA), girando em baixa velocidade sob irrigação constante a partir da superfície externa do bloco de resina composta em direção a coroa, perpendicularmente ao longo eixo do dente, no qual foram realizados seis secções para a obtenção de cinco fatias, com aproximadamente $1,0 \mathrm{~mm}$, de espessura cada que foram mantidos sob umidade relativa num frasco fechado com algodão embebido em água destilada/deionizada.

Para os espécimes submetidos ao ensaio mecânico de resistência à microtração, foram realizadas constrições na área adesiva de aproximadamente $1 \mathrm{~mm}^{2}$, obtendo, desta forma, corpos de prova com o formato de "Hour Glass" (ou ampulheta) através de pontas diamantadas extrafinas modelo 3195 FF (KG Sorensen Ind. e Com. Ltda., Barueri, SP, Brasil) montada em turbina de alta rotação extra torque (Kavo do Brasil S.A Ind. e Com. Ltda, Joinville, SC, Brasil) sob refrigeração constante.

Após a obtenção dos espécimes, os mesmos foram isolados com duas camadas de verniz para unhas de secagem rápida (Revlon, Nail Enamel 1/2Oz / 14,7 ml), sendo que, nos espécimes com o formato "Hour Glass", foi deixado uma área exposta de 2,0 $\mathrm{mm}^{2}$ de dentina e nos espécimes com o formato de disco, uma área de 3,0 $\mathrm{mm}^{2}$ de dentina adjacentes à interface de união. Ambas as áreas foram deixadas expostas dos dois lados dos espécimes para contatar diretamente com as soluções de ciclagem e o NaF.

Ciclagem dinâmica de pH (ciclo DES/RE): cada espécime apresentava uma área exposta total de $22 \mathrm{~mm}^{2}$ de dentina foi submetida à ciclagem dinâmica de $\mathrm{pH}$. O volume de solução foi calculado em função da área exposta, onde se utilizou um total de $137,5 \mathrm{ml}$ de solução DES $\left(6,25 \mathrm{ml} / \mathrm{mm}^{2}\right)$ e $68,75 \mathrm{ml}$ de solução RE $(3,125$ $\left.\mathrm{ml} / \mathrm{mm}^{2}\right){ }^{11}$

Duzentos espécimes obtidos de 40 dentes participaram do ciclo DES/RE, sendo cada ciclo correspondente a um período de 24h. Inicialmente, os espécimes foram imersos em solução desmineralizadora por um período de $30 \mathrm{~min}$. Em seguida, os mesmos foram lavados em água destilada e deionizada, secados com papel absorvente e imersos em solução aquosa de $\mathrm{NaF}$ 0,05\% por $1 \mathrm{~min}$. Após este período, os mesmos foram armazenados em solução remineralizadora por $23 \mathrm{~h}$ e $25 \mathrm{~min}$, no qual foram gastos $2 \mathrm{~min}$ para a realização das etapas anteriormente citadas de lavagem e secagem dos espécimes. No final dessas 23 h e 25 min em solução remineralizante, os espécimes foram novamente lavados em água destilada e deionizada e secados com papel absorvente, por mais 2 minutos, completando, dessa forma, o equivalente a um ciclo (24h).

Ao fim de cada cinco ciclos DES/RE, as amostras foram imersas em nova solução remineralizadora por 2 dias (48h) antes de iniciar uma nova sequência de ciclagem DES/RE por mais 5 dias. A cada 5 dias (cinco ciclos), ambas as soluções (desmineralizadora e remineralizadora) foram substituídas por novas soluções e a solução remineralizadora utilizada por $48 \mathrm{~h}$ também foi substituída no final deste tempo. O armazenamento das amostras nas respectivas soluções foi mantido a uma temperatura de $37^{\circ} \mathrm{C}$ por 7 ou 30 dias.

Foi realizada ciclagem dinâmica de $\mathrm{pH}$ (desmineralização e remineralização), para simular situações in vivo e para verificar o efeito do flúor no processo de remineralização.

Armazenamento em água destilada: a segunda metade das amostras, perfazendo um total de 200 espécimes, foi armazenada em água destilada a $37^{\circ} \mathrm{C}$ por 7 ou 30 dias, sendo a mesma trocada diariamente.

\section{- Ensaio Mecânico de Resistência à Microtração}

A sequência de fratura dos corpos de prova foi realizada de forma aleatória utilizando quatro espécimes, cada corpo de prova foi individualmente fixado pelas suas extremidades nas garras de um dispositivo específico para o teste de microtração (Bencor Multi -T, Danville Engineering Co.). O aparato foi acoplado à Máquina Universal de Ensaio (Instron 4411, Corona, Ca, USA) e o ensaio foi conduzido com uma célula de carga (Serial $N^{\circ}$ - UK 222, weight $1,2 \mathrm{Kg}$, Bolt Torque $25 \mathrm{~nm}, \pm 500 \mathrm{~N}$ ), com velocidade de $0,5 \mathrm{~mm} /$ min, até a ruptura dos espécimes. A carga necessária para a fratura de cada corpo de prova, em quilograma-força (kgf), foi anotada e as dimensões da interface adesiva do espécime foram aferidas com auxílio de um paquímetro digital (Utustools professional MT-00855, USA) para o cálculo da área. A área de cada espécime foi convertida de milímetro quadrado para centímetro quadrado e resistência à fratura em Mega Pascal (MPa) foi calculada.

\section{- Avaliação da Infiltração com Solução de Nitrato em MEV}

As fatias em forma de disco não cicladas foram isoladas com fita autocolante (Norton) e receberam duas camadas de verniz para unhas, e as anteriormente submetidas à ciclagem de $\mathrm{pH}$ receberam uma nova camada deste verniz a fim de isolar a dentina e deixar uma margem de, aproximadamente, 1,0 mm de substrato desnudo adjacente à interface adesiva. Com o intuito de evitar uma possível desidratação da dentina e uma maior infiltração da solução traçadora gerando um artefato de técnica, foi utilizado um secante instantâneo de verniz para unhas (Secante Aspa de esmalte-instantâneo, Arojet Química, Ind. Ltda., Rio de Janeiro, 
RJ, Brasil) a fim de diminuir o tempo de secagem do mesmo e submersos em solução de nitrato de prata amoniacal por $24 \mathrm{~h}$ a $37^{\circ} \mathrm{C}$. Posteriormente, as amostras foram lavadas em água corrente por 2 min, reveladas em solução reveladora (Kodak- Revelador D-76- Kodak Brasileira, Ind. e Com. Ltda, São José dos Campos, SP, Brasil) por 8h, utilizando-se iluminação fluorescente direta e indireta e fixadas em solução de Karnovisky por 1h, tamponados em cacodilato de sódio e devidamente lavados em água destilada.

\section{- Análise em MEV da Interface Adesiva}

Os espécimes foram embutidos em resina epóxica (BUEHLER Worldwide Headquarters 41 Waukegan Rd. P.O. Box 1Lake Bluff, IL 60044-1699, USA) utilizando-se, para isto, um cano de PVC (Tigre) de 2,5 cm de diâmetro isolado com vaselina sólida e fita dupla face (3M do Brasil- Sumaré, SP, Brasil), no qual os espécimes foram fixados. As amostras obtidas foram lixadas sequencialmente com lixa SiC 600, 1200 e 2400 (BUEHLER Worldwide Headquarters 41 Waukegan Rd. P.O. Box 1Lake Bluff, IL 600441699, USA) por 5 min em cada lixa e polidas com disco de feltro e pasta diamantada (Arotec Ind. e Com. Ltda, SP, Brasil) em granulação decrescente $(6,3,1$ e 0,25 $\mu \mathrm{m})$. Entre cada granulação de lixa e de pasta, as amostras foram imersas em água destilada e colocadas em ultrassom (Ultrasound Ultrason 1440 D - Odontobrás Ind. e Com. Med. Odont. Ltda, Rio Preto, SP, Brasil) por $10 \mathrm{~min}$ para remoção dos detritos. Após a limpeza das amostras, as mesmas foram secadas com papel absorvente e parte dos espécimes incluídos receberam uma gota $(0,05 \mathrm{ml})$ de ácido fosfórico a $85 \%$ por $30 \mathrm{~s}$ para remoção da matriz inorgânica dentinária e, depois, desproteinizados, utilizando-se solução de hipoclorito de sódio a $10 \%$ por $10 \mathrm{~min}$, lavados e deixados a secar a temperatura ambiente, com objetivo de expor os prolongamentos resinosos para observação em MEV do grau de infiltração do nitrato de prata nos "tags" de resina no interior dos túbulos dentinários.

A outra parte dos espécimes não recebeu o tratamento com ácido e $\mathrm{NaOCl}$. Posteriormente, as amostras foram desidratadas em álcool etílico absoluto (etanol- IUPAC) em concentrações crescentes (50, 70, 90, e 100\%) por 20 min em cada concentração e cobertas com carbono (Bal-Tec- SCD 050-Sputter Coater) para serem observadas em MEV (JEOL JSM- 5600L V), operando em alto vácuo numa potência de $20 \mathrm{KV}$, no qual foram obtidas imagens em elétrons secundários e retroespalhados ("Backscatterd Electrons”). Os espécimes infiltrados com nitrato de prata amoniacal foram submetidos à análise qualitativa através de espectometria de energia dispersiva de raios-X (EDS).

Para calcular a área infiltrada por prata nas microscopias, foi utilizado o software de computador Image J, no qual foi possível comparar a infiltração de prata após os diferentes tempos e meios de armazenamento.

\section{Resultados}

\section{- Ensaio Mecânico de Resistência à Microtração}

Os resultados obtidos no ensaio mecânico de resistência à microtração foram submetidos à análise exploratória dos dados, usando o procedimento PROC LAB do programa estatístico SAS, que indicou um "outlier" (valor discrepante no adesivo- CLSEB; tempo- 7 dias; meio- água; corpo de prova- 2), no qual foi realizada a transformação logarítmica para que os dados atendessem as pressuposições da Análise de Variância. Atendidas as pressuposições, os dados foram submetidos à Análise de Variância (ANOVA) em esquema fatorial 2 × 2 × 2 (adesivo $\times$ tempo $\times$ meio), adotando nível de significância de 5\% apresentada na Tabela 3 .

Tabela 3. Média e desvio padrão da resistência à microtração em MPa

\begin{tabular}{|c|c|c|c|}
\hline \multirow[t]{2}{*}{ Meio } & \multirow[t]{2}{*}{ Tempo } & \multicolumn{2}{|c|}{ Sistema adesivo } \\
\hline & & ASBMP (1) & CLSEB (2) \\
\hline \multirow{2}{*}{ Água (1) } & 7 dias (1) & $\begin{array}{l}\text { G1- 14,86 ( } \pm \\
5,64) \mathrm{Aa}\end{array}$ & $\begin{array}{l}\text { G5-13,16 }( \pm \\
5,26) \mathrm{Aa}\end{array}$ \\
\hline & 30 dias (2) & $\begin{array}{l}\mathrm{G} 2-13,60( \pm \\
3,57) \mathrm{Aa}\end{array}$ & $\begin{array}{l}\mathrm{G} 6-6,55( \pm \\
1,82) \mathrm{Bb}\end{array}$ \\
\hline \multirow{2}{*}{ DES/RE (2) } & 7 dias (1) & $\begin{array}{l}\text { G3- 11,86 ( } \pm \\
4,57) \mathrm{Aa}\end{array}$ & $\begin{array}{l}\text { G7- 10,27 ( } \pm \\
3,87) \mathrm{Aa}\end{array}$ \\
\hline & $30 \operatorname{dias}(2)$ & $\begin{array}{l}\text { G4- 13,24 ( } \\
4,19) \mathrm{Aa}\end{array}$ & $\begin{array}{l}\text { G8- 8,42 }( \pm \\
1,69) \mathrm{Ba}\end{array}$ \\
\hline
\end{tabular}

Observações: (1) Médias seguidas de letras distintas (maiúsculas na horizontal e minúsculas na vertical comparando tempo dentro de cada meio) diferem entre si pela ANOVA, $p<0,05$; (2) não houve diferença significativa entre os meios $(p=0,6175)$.

A Análise de Variância permitiu concluir que no meio 1 (água) e tempo 1 (7dias) os sistemas adesivos não apresentaram diferença estatística significativa (G1 e G5), porém no mesmo meio 1 (água) e tempo 2 (30 dias) os sistemas adesivos diferiram estatisticamente entre si (G2 e G6). Para o meio 2 (DES/RE) no tempo 1 (7dias) não houve diferença estatística significativa entre os adesivos (G3 e G7), diferente do que ocorreu no mesmo meio 2 (DES/RE) mas no tempo 2 (30 dias), em que os adesivos apresentaram diferença estatística significativa (G4 e G8).

Quando se considerou o fator sistema adesivo, o resultado da ANOVA permitiu concluir que para o sistema adesivo ASBMP em água não houve diferença estatística significativa entre os tempos de 7 ou 30 dias. Neste mesmo meio, para o sistema adesivo CLSEB houve diferença estatística significativa entre os tempos de armazenamento. Em contrapartida, os grupos submetidos ao processo DES/RE não demonstraram diferença estatística significativa para ambos os adesivos nos dois tempos avaliados.

\section{Microinfiltração}

\section{- Adper Scotchbond Multi-Purpose}

Após 7 dias de armazenamento, independente da solução utilizada, as ilustrações demonstraram um pequeno acúmulo de partículas de prata na base da camada híbrida (figura 1). Após 30 dias de armazenamento dos espécimes, as ilustrações microscópicas e a análise da área infiltrada por corante a base de prata demonstraram um maior acúmulo dessas partículas (figura 2), em comparação ao tempo de 7 dias. No entanto, a ciclagem dinâmica de $\mathrm{pH}$ por 30 dias reduziu a área infiltrada. A figura 3 ilustra análises de EDS para este sistema adesivo. 


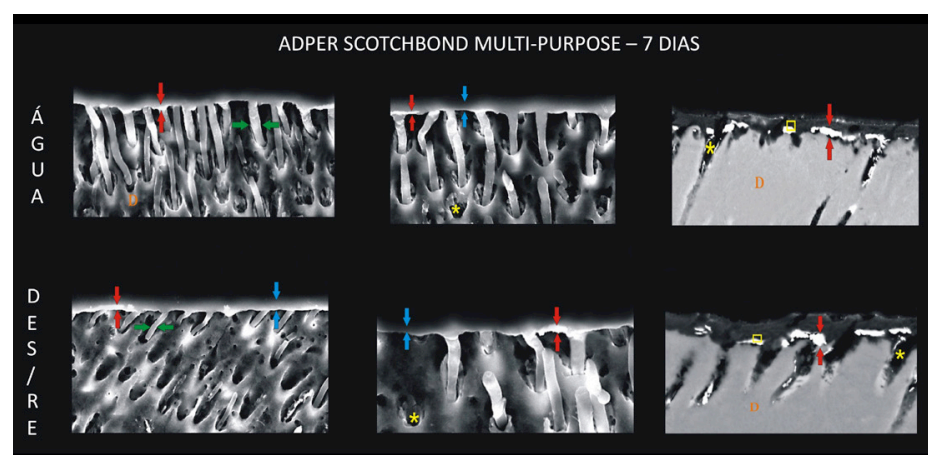

Figura 1. Fotomicrografias (1.500x) ilustrando interface adesiva formada pelo sistema Adper Scotchbond Multi-Purpose após 7 dias de armazenamento. Seta azul indicando camada híbrida; seta vermelha indicando com presença de prata; seta verde indicando tag resinoso; * indicando túbulo dentinário

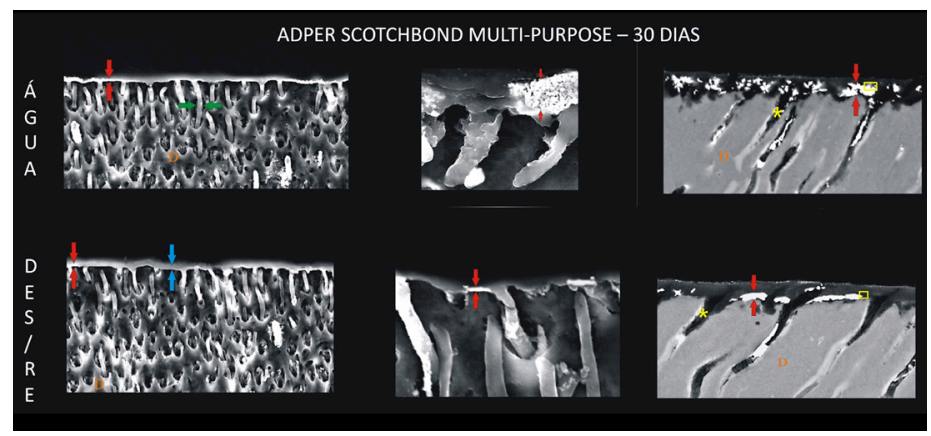

Figura 2. Fotomicrografias (1.500x) ilustrando interface adesiva formada pelo sistema Adper Scotchbond Multi-Purpose após 30 dias de armazenamento. Seta azul indicando camada híbrida; seta vermelha indicando com presença de prata; seta verde indicando tag resinoso; * indicando túbulo dentinário

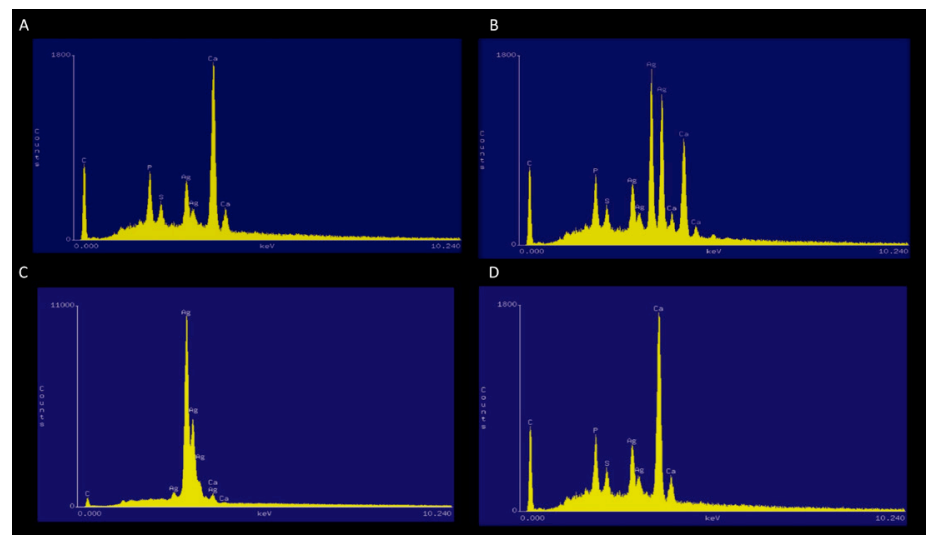

Figura 3. EDS das amostras referente ao sistema Adper Scotchbond Multi-Purpose, após 7 dias de armazenamento em água (A) e solução DES/RE (B) e após 30 dias de armazenamento em água (C) e solução DES/RE (D)

\section{- Clearfil SE Bond}

Após 7 dias de armazenamento em água, foi observado um característico infiltrado de partículas de prata na base da camada híbrida. Neste mesmo período, independente do meio de estocagem, as ilustrações demonstraram um semelhante pequeno acúmulo dessas mesmas partículas (figura 4). A presença de poros infiltrados por prata no interior da camada híbrida, aumentou progressivamente de 7 para 30 dias de armazenamento em água. No entanto, as imagens obtidas em MEV de amostras desgastadas com a emissão de elétrons retroespalhados e submetidas à microanálise de raios-X (EDS), identificou a presença de prata com picos atômicos para moléculas ricas em cálcio e fosfato no interior da camada híbrida, apresentando uma menor infiltração de prata, quando utilizado o sistema Clearfil SE Bond armazenado em solução DES/RE mais NaF por 30 dias (Figuras 5 e 6).

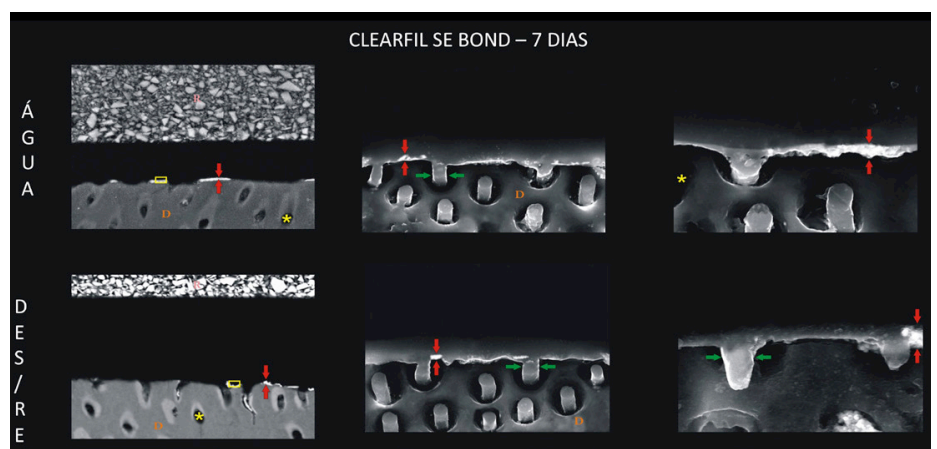

Figura 4. Fotomicrografias (3.500x) ilustrando interface adesiva formada pelo sistema Clearfil SE Bond, após 7 dias de armazenamento. Seta azul indicando camada híbrida; seta vermelha indicando com presença de prata; seta verde indicando tag resinoso; * indicando túbulo dentinário

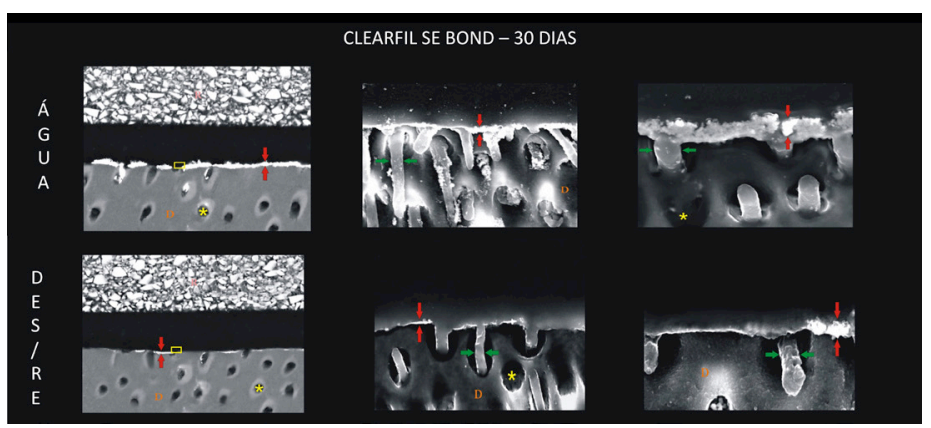

Figura 5. Fotomicrografias (3.500x) ilustrando interface adesiva formada pelo sistema Clearfil SE Bond, após 7 dias de armazenamento. Seta azul indicando camada híbrida; seta vermelha indicando com presença de prata; seta verde indicando tag resinoso; * indicando túbulo dentinário

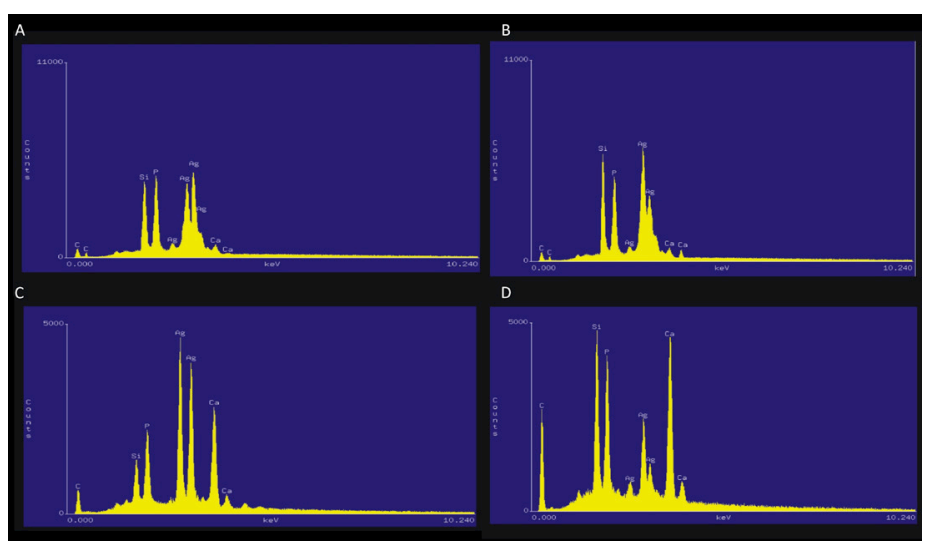

Figura 6. EDS das amostras referente ao sistema Clearfil SE Bond; após 7 dias de armazenamento em água (A) e solução DES/RE (B) e após 30 dias de armazenamento em água (C) e solução DES/RE (D)

\section{Discussão}

A ciclagem dinâmica de $\mathrm{pH}$ é um dos métodos mais respeitados e consagrados para avaliar o comportamento de substratos dentais sobre eventos de desmineralização e remineralização, no qual se utilizam soluções balanceadas a partir da manipulação de diferentes concentrações de sais e ácidos, possibilitando mi- 
metizar uma situação mais próxima do processo físico-químico dinâmico, com trocas minerais entre tecidos duros dentais e fluidos bucais. ${ }^{12}$ Devido às inúmeras pesquisas na área de adesão à dentina que levaram a elucidação da contribuição de cada um dos componentes destes materiais, altos valores de resistência adesiva inicial têm sido alcançados, porém a estabilidade e, consequentemente, a durabilidade da adesão continuam sendo uma incógnita tendo visto que os componentes hidrofílicos, como o HEMA, que compõe o adesivo e possibilitam sua infiltração no tecido dentinário para formação de uma camada híbrida satisfatória, apresentam a estrutura química sujeita a hidrólise a longo prazo. ${ }^{13,14}$

Neste estudo, a hipótese avaliada foi verificar "in vitro" se o tratamento químico dinâmico de $\mathrm{pH}$, mimetizando as condições iônicas dos minerais presentes na saliva, sem induzir os espécimes à cárie, seria capaz de remineralizar, junto à utilização de solução fluoretada, os poros no interior e áreas subjacentes à camada híbrida através da formação de fosfato de cálcio na forma de hidroxiapatita e fluorapatita após 30 dias de armazenamento, comparando com o armazenamento dos espécimes pelo mesmo tempo em água, através da resistência de união e de forma ilustrativa da interface adesiva infiltrada com solução de nitrato de prata.

Além disso, estudos indicam uma grande possibilidade do uso de fluoretos apresentar uma capacidade inibitória sobre as metaloproteinases, ${ }^{15}$ enzimas capazes de degradar as fibras colágenas presente no tecido da dentinário em áreas nas áreas onde as mesmas não se apresentam completamente embebidas pelo sistema adesivo, este processo é ativado pela redução do $\mathrm{pH}$ causada pelo condicionamento ácido da técnica adesiva. ${ }^{16,17}$

Foi observado que as amostras tratadas com o sistema adesivo Clearfil SE Bond apresentaram valores de resistência adesiva significativamente maiores quando os espécimes foram ciclados e tratados com $\mathrm{NaF}$ por 30 dias em comparação aos armazenados em água pelo mesmo período de tempo, que sofreram queda significativa da adesão. No entanto, quando os mesmos foram armazenados por um curto período de tempo (7 dias) em solução DES/ RE ou em água, não apresentaram diferença estatística significativa do grupo ciclado por 30 dias.

Apesar da pouca espessura da camada híbrida constituída pela "smear layer" e dentina modificada formada por sistemas autocondicionantes, uma menor quantidade de defeitos é incluída no seu interior devido à ausência de discrepâncias entre a profundidade de desmineralização e a profundidade de penetração do adesivo no aderente. Isto resulta na obtenção de satisfatórios valores iniciais de adesão como os observados neste trabalho. ${ }^{8,18}$

Como estes sistemas adesivos se propõem a formar uma zona de interdifusão mais uniforme, era de se esperar uma manutenção constante dos valores de adesão após diferentes períodos de armazenamento em água. Porém, o contrário foi observado, quando os valores de adesão apresentados pelo sistema Clearfil SE Bond, após o mesmo ter sido armazenado por 30 dias em água, decresceu de maneira significante, o que pode ser resultante do fato dos adesivos autocondicionantes apresentarem um maior percentual de componente hidrofílico com a finalidade de aumentar a capacidade de molhabilidade destes compostos sobre a dentina possibilitando a ação dos monômeros ácidos que promovem seu condicionamento.

Os componentes hidrofílicos fazem com que estes sistemas se comportem como uma membrana semipermeável com elevada capacidade de sorção de água que pode acarretar perdas significativas das propriedades seja pela quebra de cadeia por hidrólise ou pela sua plastificação que reduz intensamente a resistência coesiva da matriz que compõe estes materiais. ${ }^{14}$ Além disso, incorporação da "smear layer" no complexo híbrido resulta na formação de um elo fraco na adesão, pois seu aspecto extremamente poroso facilita a sua dissolução, o que causa um aumento do potencial de infiltração de fluidos e produtos bacterianos nos espaços deixados pela mesma, ${ }^{8,18}$ uma vez que, os cristais de hidroxiapatita parcialmente dissolvidos, leva a formação de espaços nanométricos entre as fibras colágenas, nos quais são encontrados subprodutos da reação, que agem como filtros moleculares ${ }^{19}$ que representam outra contribuição para a degradação da união ao longo do tempo.

Por conseguinte, a conjunção destes fatores culmina na degradação em longo prazo da camada formada por destes adesivos conforme observado através das ilustrações obtidas em MEV utilizando o sistema Clearfil SE Bond que após 7 dias de armazenamento em água, já apresentou um infiltrado de partículas de prata na base da camada híbrida. A confirmação deste processo de degradação também foi confirmada através de uma diminuição significativa dos valores de adesão e a maior infiltração de partículas de prata após o armazenamento por 30 dias quando comparado ao armazenamento por 7 dias em água.

Entretanto, o tratamento químico dinâmico de $\mathrm{pH}$, associado à imersão em solução fluoretada avaliados após 30 dias de armazenamento, proporcionou uma estabilização nos valores de resistência adesiva, quando comparado aos valores iniciais de adesão produzidos pelo mesmo sistema, possivelmente causada pela deposição de minerais nos poros presentes na interface adesiva, alterando a composição estrutural dessa camada,20 além de possibilitar a provável interação química desses monômeros, presentes na composição destes sistemas, com o cálcio, precipitado pela ação da ciclagem química dinâmica de $\mathrm{pH}$, fundindo-se com a dentina mineralizada, retardando, desta forma, o processo de degradação da interface de união. ${ }^{21}$

Alguns autores, ${ }^{21}$ em 2001 realizaram um estudo "in vivo", no qual foi possível identificar através de microanálise de raios-X a possível remineralização de espaços nanométricos abaixo da camada híbrida após 6 meses das restaurações confeccionadas "in vivo”. Neste estudo identificou-se a presença de prata com picos atômicos para moléculas ricas em cálcio e fosfato na camada híbrida, com uma menor infiltração calculada para o corante prata, quando utilizado o sistema Clearfil SE Bond, armazenado em solução DES/RE mais NaF por 30 dias.

Uma união estável, conseguida através da formação de uma camada híbrida densa é fator primordial para durabilidade da união. A literatura, em sua grande maioria, demonstra através de estudos longitudinais que adesivos de três passos são utilizados como referência para se testar a adesão, por apresentarem uma união mais estável ao se comparar aos de passo único autocondicionantes ou de dois passos convencionais. ${ }^{22}$ Corroborando com estes resultados este estudo demonstrou, que o sistema adesivo Adper Scotchbond Multi-Purpose apresentou estabilidade de união independente do tempo e solução de armazenamento, não apresentando diferença estatística significativa nos valores de adesão. Em contrapartida, as ilustrações microscópicas e a análise da interface infiltrada por prata demonstraram um pequeno, porém, maior acúmulo de prata na camada híbrida, retratando um ligeiro, mas presente processo de degradação após seu armazenamento em água por 30 dias.

O pequeno processo de degradação da interface adesiva observada no sistema três passos pode ter sido decorrente principalmente de áreas que continham monômeros não convertidos que foram lixiviados da estrutura deixando lacunas nesta área ou por espaços que foram desmineralizados, mas não preenchidos de adesivo por sua inabilidade de chegar por capilaridade até a 
região. Por conseguinte, a ciclagem dinâmica de $\mathrm{pH}$ por 30 dias reduziu a infiltração do agente traçador utilizado, no fato ligado a provável deposição de minerais nestes espaços nanométricos existentes em meio a interface.

Diante do exposto pode-se concluir que parte da camada híbrida que serviriam de pontos de partida para a degradação da interface adesiva pode ser reparada ao longo do tempo com a utilização de substâncias que contenham alta concentração de flúor, retardando clinicamente o processo de degradação da interface adesiva, através da deposição de minerais nas áreas de espaços livres dessa estrutura.

\section{Conclusão}

De acordo com os fatores avaliados neste estudo e considerando os resultados obtidos, conclui-se que: ambos os sistemas adesivos estudados (Clearfil SE Bond e Adper Scotchbond Multi-Purpose) apresentaram resistência de união inicial satisfatória (armazenados em água por 7 dias), não apresentando diferença estatística significativa; houve um decréscimo nos valores da resistência de união significativo quando o sistema Clearfil SE Bond foi estocado por 30 dias em água. Entretanto, a utilização do sistema Adper Scotchbond Multi-Purpose não sofreu alteração significativa nos dois períodos de armazenamento; o armazenamento dos espécimes hibridizados com o sistema Clearfil SE Bond por 30 dias em solução DES/RE, associado à imersão em NaF 0,05\%, aumentou os valores de resistência de união em comparação a 30 dias de armazenamento em água. No entanto, o armazenamento dos espécimes hibridizados com o sistema Adper Scotchbond Multi-Purpose não apresentou diferença estatística significativa nos valores de resistência de união em ambos os tempos de armazenamento; independente do tempo e do meio de armazenamento, as microscopias ilustraram um padrão de nanoinfiltração quando ambos os sistemas adesivos foram utilizados.

\section{Referências}

1. Rosa WL, Piva E, Silva AF. Bond strength of universal adhesives: A systematic review and meta-analysis. J Dent. 2015;43(7):765-76.

2. Tjäderhane L, Nascimento FD, Breschi L, Mazzoni A, Tersariol IL, Geraldeli S, et al. Strategies to prevent hydrolytic degradation of the hybrid layer-A review. Dent Mater. 2013;29(10):999-1011.

3. Van Landuyt KL, Snauwaert J, De Munck J, Peumans M, Yoshida Y, Poitevin A, et al. Systematic review of the chemical composition of contemporary dental adhesives. Biomaterials. 2007;28(26):3757-85.

4. Van Landuyt KL, Snauwaert J, De Munck J, Coutinho E, Poitevin A, Yoshida Y, et al. Origin of interfacial droplets with one-step adhesives. J Dent Res. 2007;86(8):739-44.

5. Tjäderhane L, Nascimento FD, Breschi L, Mazzoni A, Tersariol IL, Geraldeli S, et al. Optimizing dentin bond durability: control of collagen degradation by matrix metalloproteinases and cysteine cathepsins. Dent Mater. 2013;29(1):116-35.

6. Carvalho AO, Bacelar-Sá R, Wodevotzky Jr. O, Ambrosano GMV, Magne PM, et al. Bond strength and micromorphology of resin-dentin interface of etch-and-rinse dentin bonding agents after 1-year of water storage. Appl Adhes Sci. 2016;4(16):1-8. 7. Breschi L. Dental adhesion review: Aging and stability of the bonded interface. Dent Mater. 2008;24(1):90-101.

8. Tay FR, Sano H, Carvalho R, Pashley EL, Pashley DH. An ultrastructural study of the influence of acidity of self-etching primers and smear layer thickness on bonding to intact dentin. J Adhes Dent. 2000;2(2):83-98.

9. Pereira GD, Paulillo LA, De Goes MF, Dias CT. How wet should dentin be? Comparison of methods to remove excess water during moist bonding. J Adhes Dent. 2001;3(3):257-64.

10. Nalçaci A, Salbaş M, Ulusoy N. The effects of soft-start vs continuous-light polymerization on microleakage in Class II resin composite restorations. J Adhes Dent. 2005;7(4):309-14.

11. Shinkai RS, Del Bel Cury AA, Cury JA. In vitro evaluation of secondary caries development in enamel and root dentin around luted metallic restoration. Oper
Dent. 2001;26(1):52-9.

12. Maior JR, Da Figueira MA, Netto AB, de Souza FB, da Silva CH, Tredwin CJ. The importance of dentin collagen fibrils on the marginal sealing of adhesive restorations. Oper Dent. 2007;32(3):261-5.

13. Torkabadi S, Nakajima M, Ikeda M, Foxton RM, Tagami J. Bonding durability of HEMA-free and HEMA-containing one-step adhesives to dentine surrounded by bonded enamel. J Dent. 2008;36(1):80-6.

14. Takahashi M, Nakajima M, Hosaka K, Ikeda M, Foxton RM, Tagami J. Longterm evaluation of water sorption and ultimate tensile strength of HEMA-containing/-free one-step self-etch adhesives. J Dent. 2011;39(7):506-12.

15. Donmez N, Belli S, Pashley DH, Tay FR. Ultrastructural correlates of in vivo/in vitro bond degradation in self-etch adhesives. J Dent Res. 2005;84(4):355-9.

16. Carrilho MR, Carvalho RM, de Goes MF, di Hipólito V, Geraldeli S, Tay FR, et al. Chlorhexidine preserves dentin bond in vitro. J Dent Res. 2007;86(1):90-4.

17. Manfro AR, Reis A, Loguercio AD, Imparato JC, Raggio DP. Effect of different concentrations of chlorhexidine on bond strength of primary dentin. Pediatr Dent. 2012;34(2):e11-5.

18. Tay FR, Carvalho R, Sano H, Pashley DH. Effect of smear layer on the bonding of self-etching primer to dentin. J Adhes Dent. 2000;2(2):99-116.

19. Carvalho RM, Chersoni S, Frankenbergr R, Pashley DH, Prati C, Tay FR. A challenge to the convencional wisdom that simultaneous etching and resin infiltration always occurs in self-etch adhesives. Biomaterials. 2005;26(9):1035-42.

20. Pereira GDS, Paulillo LAMS. Influence of dentin moisture on adhesive system's shear bond strength. J Dent Res. 2000;79(2392):442 (Special Issue).

21. Akimoto N, Yokoyama G, Ohmori K, Suzuki S, Kohno A, Cox CF. Remineralization across the resin-dentin interface: in vivo evaluation with nano in dentation measurements, EDS, and SEM. Quintessence Int. 2001;32(7):560-1.

22. Cardoso MV, de Almeida Neves A, Mine A, Coutinho E, Van Landuyt K, De Munck J, et al. Current aspects on bonding effectiveness and stability in adhesive dentistry. Aust Dent J. 2011;56 Suppl 1:31-44.

\section{Mini Currículo e Contribuição dos Autores}

1. Leonardo Vieira de Oliveira - cirurgião-dentista e mestre em Clínica Odontológica. Contribuição: desenvolvimento da parte experimental.

2. Maíra do Prado - cirurgiã-dentista e PhD em Clínica Odontológica. Contribuição: redação do artigo.

3. Carlos Tadeu dos Santos Dias - estatístico e professor titular ESALQ- USP. Contribuição: análise estatística e interpretação dos resultados.

4. Adriano Nóbrega de Castro - cirurgião-dentista e especialista em Endodontia. Contribuição: desenvolvimento da parte experimental.

5. Gisele Damiana da Silveira Pereira - cirurgiã-dentista e PhD em Clínica Odontológica. Contribuição: interpretação dos resultados e revisão crítica.

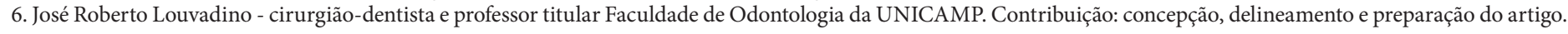

\section{Autor Correspondente}

Maíra do Prado

E-mail: mairapr@hotmail.com 